\title{
BMJ Open Older patient considering treatment for advanced renal disease: protocol for a scoping review of the information available for shared decision-making
}

\author{
Rajesh Raj, ${ }^{1}$ Kiran D K Ahuja, ${ }^{2}$ Mai Frandsen, ${ }^{2}$ Matthew Jose ${ }^{1}$
}

To cite: Raj R, Ahuja KDK, Frandsen $\mathrm{M}$, et al. Older patient considering treatment for advanced renal disease: protocol for a scoping review of the information available for shared decision-making. BMJ Open 2016;6:e013755. doi:10.1136/bmjopen-2016013755

- Prepublication history and additional material is available. To view please visit the journal (http://dx.doi.org/ 10.1136/bmjopen-2016013755).

Received 4 August 2016 Revised 22 October 2016 Accepted 15 November 2016

CrossMark

\footnotetext{
${ }^{1}$ School of Medicine, University of Tasmania, Launceston, Tasmania, Australia

${ }^{2}$ School of Health Sciences, University of Tasmania, Launceston, Tasmania, Australia
}

Correspondence to Dr Rajesh Raj; rajesh.raj@ths.tas.gov.au

\begin{abstract}
Introduction: Older adults constitute the largest group of patients on dialysis in most parts of the world. Management of advanced renal disease in the older adult is complex; treatment outcomes and prognosis can be markedly different from younger patients. Clinical teams caring for such patients are often called on to provide information regarding prognosis and outcomes with treatmentparticularly, the comparison between having dialysis treatment versus not having dialysis. These discussions can be difficult for clinicians because they have to contend with incomplete or nascent data regarding prognosis and outcomes in this age group. We aim to summarise the currently available information regarding the prognosis and outcomes of advanced renal disease in the older adult by means of a scoping review of the literature. This article discusses our protocol.
\end{abstract}

Methods: This scoping review will be undertaken in accordance with the Joanna Briggs Institute's methodology for scoping reviews. A directed search will look for relevant articles in English (within electronic databases and the grey literature), written between 2000 and 2016, which have studied older patients with advanced renal disease (estimated glomerular filtration rate $<30 \mathrm{~mL} / \mathrm{min} / 1.73 \mathrm{~m}^{2}$ ). After screening by two independent reviewers, selected articles will be analysed using a data charting tool. Reporting will include descriptions, analysis of themes using qualitative software and display of information using charts.

Ethics and dissemination: This scoping review will analyse previously collected data, and so does not require ethical approval. Results will be disseminated through academic journals, conferences and seminars. We anticipate that our summary of the currently available knowledge regarding the older adult with advanced renal disease will be a repository of information for clinicians in the field. We expect to identify areas of study that are suited to systematic reviews. Our findings can also be expected to influence guidelines and clinical practice recommendations in the future.

\section{Strengths and limitations of this study}

- Previous reviews have not attempted to systematically collect, describe and synthesise all the considerations in making treatment decisions for the older adult with advanced renal disease. This review will collect information across quantitative and qualitative spectra of research, drawing on both published and grey literature. It will also describe data that highlights patient and carer perspectives, both of which are relevant to this life-intrusive illness and its treatment. This widens the sources of information beyond those traditionally used for systematic reviews in the area - this will be a strength of the proposed study.

- Such a wide-ranging review can serve as a useful repository of information for clinicians and others working (or conducting research) in this area; it can suggest areas for further systematic reviews and contribute to generating guidelines.

- The scoping review conducted according to this protocol will address the information available for the shared decision-making process in the older adult. So as to preserve focus, it does not include other aspects of dialysis decisionmaking, including how this information is presented/received, types of decision-making models, decision science, the impact of health literacy, socioeconomic factors, mental capacity and cognition, cultural/language barriers or resource limitations.

- Studies included will not undergo a formal quality assessment-this is part of the design, as a scoping review attempts to describe all the information available, rather than only select the highest quality of evidence.

- This protocol is for a scoping review that only considers material written in English. Potentially, large populations of the non-English-speaking world may not be represented. Our conclusions may not apply to the different cultural and social environments in these regions. 


\section{BACKGROUND}

Approximately half of all patients on dialysis in Australia at the end of 2014 were aged 65 and above. Patients aged 65-85 years have the highest incidence (patients per million) of renal replacement therapies. ${ }^{1}$ These numbers will conceivably rise in subsequent years as the population on dialysis ages, and as incident patients are added. However, several researchers have suggested that the older patient may not do well on dialysis in terms of quality of life, preservation of independence or survival. Studies suggest that in the presence of severe comorbidities such as frailty or heart disease, there is no survival advantage to being on dialysis. ${ }^{2}$ On the contrary, some older patients who choose not to have dialysis enjoy a good quality of life, and may not have a significantly shortened survival in comparison. ${ }^{2-6}$

Several renal units now also offer a distinct, nondialysis pathway of care for patients opting not to have dialysis treatment for end-stage renal disease-thus providing another valid and available treatment choice for these patients. ${ }^{7}$ This pathway may be called the 'conservative', 'supportive' or 'renal palliative' care pathway.

Principles of holistic care in the management of the older patient are widely applicable-including detailed symptom management, advance care planning, functional assessment and appropriate support, and targeted measures to improve quality of life. However, uncertainty exists regarding the benefits of dialysis therapy in the older adult. Predicting which older patient will do well on dialysis is quite difficult.

Nephrologists and other members of the renal team are often central to the discussions around treatment choices for advanced renal impairment. National organisations, such as the Australia New Zealand Society of Nephrology or the Renal Physicians Association in the USA, suggest a process of shared decision-making for patients considering dialysis. ${ }^{8}{ }^{9}$ Shared decision-making is defined as 'a process by which a healthcare choice is made by the patient (or significant others, or both) together with one or more healthcare professionals'. Clinical practice recommendations in this area suggest that 'nephrologists do not shy away from these discussions'-rather, they suggest, treating teams ought to have 'realistic discussions' with patients about survival and quality of life with and without dialysis treatment. ${ }^{8}$

The provision of information is an important component of shared decision-making, as exemplified in several popular models of the process. For instance, in the Interprofessional Shared Decision-Making Model, ${ }^{10}$ 'information exchange' is an integral part of the process. Similarly, in the model suggested by Elwyn and colleagues, ${ }^{11}$ which consists of a 3-step shared decisionmaking model for clinical practice-'choice talk, option talk and decision talk', provision of information is an integral part of discussions about options and choice. Accurate information is central to shared decisionmaking, as it 'rests upon knowing and understanding the best available evidence on the risks and benefits across all available options, while ensuring that the patient's values are taken into account'. ${ }^{10}$ However, providing or accessing such information often proves difficult for the clinician, as sufficient, comprehensive information is not readily available.

Comprehensive conservative treatment for renal impairment that does not include dialysis is an actively evolving paradigm of care, with few practices backed by high-quality evidence, making standardisation difficult. Additionally, most such care is provided in heterogeneous settings, by different professionals (eg, multidisciplinary clinics). Such factors make head-to-head comparisons of the two modalities (dialysis vs nondialysis) cumbersome, limiting the information available for a discussion comparing the two pathways.

There are other practical difficulties. Studies have shown significant variability in how different doctors make decisions about recommending dialysis. ${ }^{12}{ }^{13}$ Estimates of prognosis made by doctors are likely to be inaccurate. Age and non-renal factors may not always receive consideration. Factors such as comorbidities, frailty, mental status, dependency for transfers and residence in a nursing home can all impact on the prognosis on dialysis, but it is difficult to consider these variables systematically in making decisions. Efforts to construct prognostic indices for the older adult considering dialysis have met with limited acceptance. Often, these indices document prognosis for patients already on dialysis, or they do not consider non-renal factors. Not surprisingly, patients may consider non-medical factors important to their decision-such as the number of hospital visits required, or the restrictions on travel. ${ }^{14}{ }^{15}$ Clinicians may not be aware of such research into patient and caregiver preferences for treatment or end-of-life choices.

In summary, there are uncertainties and gaps in knowledge when renal teams are called on to provide appropriate comparisons between treatment with or without dialysis in the older patient with advanced renal disease. The life-sustaining nature of dialysis presents difficulties in the design of a randomised trial comparing dialysis treatment with treatment without dialysis in this population. Given this scenario, the scoping review methodology, extending across quantitative and qualitative research domains, appears well suited as a first step in detailing the breadth of information available in this particular area at present. From the information gathered, we anticipate that the need for future systematic reviews in particular areas will be identified.

\section{METHODS AND ANALYSIS}

(Please see figure 1 for a flow chart detailing the major steps in the scoping review.)

This scoping review will be undertaken in accordance with the Joanna Briggs Institute's methodology for scoping reviews. ${ }^{16}$ 
Preliminary List of Search Terms

Aged;Geriatric; Elderly; Older; Chronic Kidney Disease; Chronic Kidney Failure; Chronic Renal Insufficiency;

Renal Replacement Therapy: Renal Dialysis;Conservative care; Palliative care; supportive care; Withholding treatment:Risk Assessment: Risk Factors; Logistic Models; Time Factors; Life Expectancy; Kaplan-Meier Estimate; Treatment Outcome; Prognosis; Prognostic score; Quality of life; Lived Experience; Adaptation, psychological; Patient Selection; Decision Making; Information Needs; Patient Education; Patient preference; Physician advice; Doctor-patient communication; Surveys and questionnaires

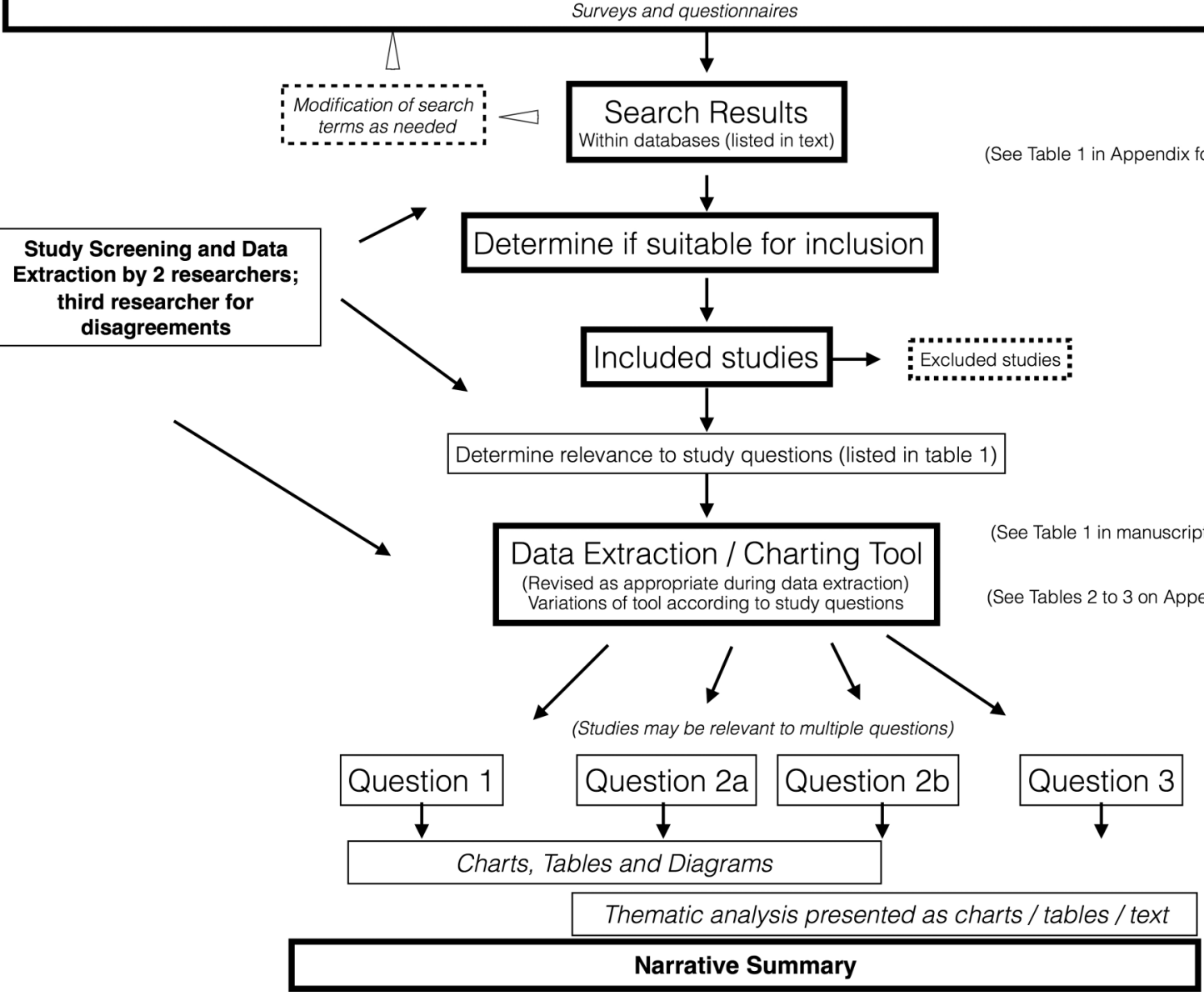

Figure 1 Flow chart of proposed scoping review.

From readings of published literature and clinical guidelines/recommendations, we anticipated, empirically, that five broad categories of information are likely to be relevant to the discussions around treatment options for the older adult, as follows:

- information about prognosis/survival in older patients with advanced renal disease managed either with or without dialysis treatment;

- information about quality of life in older patients with advanced renal disease managed either with or without dialysis treatment;

- information describing the lived experiences of the older adult with advanced renal disease;

- information on the factors important to older patients and their careers as they make treatment choices around advanced renal disease;

- other factors, not included above.

A preliminary search of the literature in The Cochrane Library, JBI Database of Systematic Reviews and Implementation Reports, TRIP database and Prospero failed to identify a scoping review that summarises the entire range of considerations discussed above.

\section{Operational definitions}

\section{Older adult}

Different chronological ages have been used in the literature to define the term 'older adult' or 'elderly'. ${ }^{17}$ In order to include all relevant data, we will include studies where the population studied has been described by primary researchers as 'elderly', 'geriatric' or 'older adult' without specifying beforehand an age cut-off to define the older adult. In the summaries created, we will mention the ages of patients included under this term in relevant studies.

\section{Patients with advanced renal disease}

This is defined as patient populations in any of the following categories:

- having established ( $>3$ months) renal impairment with an estimated glomerular filtration rate (eGFR) of $<30 \mathrm{~mL} / \mathrm{min} / 1.73 \mathrm{~m}^{2}$;

- described as having 'advanced renal disease' by the primary researchers;

- receiving education regarding renal replacement therapies; 
- on dialysis:

- on non-dialysis, supportive or conservative care.

\section{Carers}

Includes all individuals involved in directly caring for the patient, whether associated through family, friendship or marriage.

\section{Clinicians}

Clinicians include doctors, nurses and allied healthcare staff directly involved in the medical care of, and the shared decision-making process with, patients and carers.

\section{Dialysis treatment}

Dialysis treatment involves renal dialysis, including all forms of haemodialysis and peritoneal dialysis, including in-centre, home-based, assisted or self-care approaches.

\section{Conservative care}

Conservative care includes care given to patients with advanced renal disease who have decided not to undergo dialysis treatment, described as 'non-dialysis', 'supportive', 'conservative' or 'palliative' renal care.

\section{Objectives}

The objective of this review is to identify and summarise the nature and scope of information available for consideration when discussing treatment options for advanced renal disease with an older patient.

The study aims to synthesise information from quantitative and qualitative literature, so as to

- provide a coherent summary for clinicians;

- explore the need for future comprehensive systematic reviews on the subject.

\section{Review questions}

The questions for this scoping review are as follows (summarised in box 1):

- What information is available to be used in the shared decision-making process for the older adult considering treatment options for advanced renal disease? Specifically, the details examined are:

\section{Box 1 Review questions}

1. What are the factors affecting prognosis and survival in the older patient with advanced renal disease either choosing to have dialysis treatment, or choosing to have conservative care without dialysis?

2. (A) What factors influence the quality of life in the older patient being treated for advanced renal disease?

(B) What information is available regarding the lived experiences of older adults treated for advanced renal disease?

3. What is known about the information needs of older adults and their carers considering treatment options for advanced renal disease?
- markers of prognosis (survival) in the older patient with advanced renal disease;

- factors influencing quality of life in the older patient with advanced renal disease;

- reports of lived experiences of older adults undergoing treatment (with or without dialysis) for advanced renal disease.

- What do we know about the information needs of older adults and their carers as they consider treatment options for advanced renal disease?

\section{Inclusion criteria}

Study selection

This scoping review will consider, for all questions, articles that address the older adult with advanced renal disease, their carers or the clinicians involved in their care, regardless of sex, region, diagnoses or comorbidities (see figure 1). Articles from peer-reviewed scientific literature as well as those from grey literature will be considered (details below).

\section{Concept}

The core concept of this scoping review is to provide a summary of the breadth of information relevant to discussions and decision-making in the older adult with advanced renal disease who is considering treatment options. The primary focus is on the information that is likely to be of value in choosing whether to have dialysis (any type of dialysis) or not. With regard to the specific questions articulated above,

- For question 1, studies that report on prognosis, prognostic indices, survival and mortality in the population described will be considered for inclusion.

- For question 2 (A), studies that describe quality of life data in this population, either in isolation or in relationship to other variables, including descriptive/ observational and interventional studies will be included.

- For question 2 (B), studies that describe the lived experience of these patients and their carers will be considered.

- For question 3, studies that have described the information needs of older adults and their carers around the decision-making process in advanced renal disease will be considered.

\section{Context}

This scoping review will consider articles pertaining to the older adult in inpatient, outpatient, home or residential care facility settings.

\section{Sources: study types}

(Please see online supplementary table S1 for a draft version of the initial appraisal tool.)

This scoping review will consider quantitative and qualitative study designs, including: 
- Experimental and quasi-experimental studies (randomised and non-randomised controlled trials), before and after studies and interrupted time-series studies.

- Analytical and descriptive observational studies including prospective and retrospective cohort studies, case-control and cross-sectional studies, case series and case reports.

- Qualitative studies will also be considered that focus on qualitative data including, but not limited to, designs such as phenomenology, grounded theory, ethnography, qualitative description and action research.

- We will also include searches of the grey literature (see description of databases below).

- Textbook chapters and opinion papers will also be considered for inclusion.

- We will also include recommendations made by national bodies involved in setting standards and providing guidelines for renal care.

Studies published from January 2000 to October 2016 will be included so as to reflect the increasing number of older patients on dialysis, the changing attitudes to the treatment of older adults in recent years and the establishment of conservative care without dialysis as a valid treatment option. Only studies with abstracts published in English will be included in the initial screening process.

\section{Exclusion criteria}

- research that does not address older adults (see operational definition above) as a main or subpopulation of interest;

- research that exclusively addresses patients with an eGFR $>30 \mathrm{~mL} / \mathrm{min} / 1.73 \mathrm{~m}^{2}$;

- studies in languages other than English.

\section{Search strategy}

An initial limited search of MEDLINE, Scopus, Embase, PsycINFO, CINAHL and Cochrane Library databases has been undertaken to identify articles on this topic. Analysis of the words contained in the titles, abstracts and index terms used to describe these articles was used to develop an initial list of search terms and keywords, as follows:

- humans; aged; geriatric; elderly; older;

- chronic kidney disease; chronic kidney failure; chronic renal insufficiency; renal replacement therapy; renal dialysis;

- conservative care; palliative care; supportive care; withholding treatment;

- risk assessment; risk factors; logistic models;

- time factors; life expectancy; Kaplan-Meier estimate;

- treatment outcome; prognosis; prognostic score; quality of life; lived experience; adaptation, psychological;

- patient selection; decision-making; information needs; patient preference; patient education; physician advice; doctor-patient communication; surveys and questionnaires.

The keywords/search terms will be appropriately used for each database. As studies are being considered, their reference lists will be screened for additional studies.

\section{Databases searched}

The following databases will be searched: PubMed PsycINFO, CINAHL, Embase, Scopus, Mednar, Turning research into practice, NTIS, ProQuest Dissertations and Theses, Google Scholar and Current Contents.

The search for articles in the grey literature will include electronic sources including OpenSIGLE, Healthcare Management Information Consortium (HMIC) Database, National Technical Information Service (NTIS), PsycEXTRA, BIOSIS Databases, Open Grey, Trove, EThOS, OATD.org and OpenThesis.

The search for guidelines will include searches at the National Guideline Clearinghouse, http://www.cari.org, http://www.kdigo.org, the National Kidney Foundation (NKF-DOQI), Kidney Health Australia, the Renal Physicians Association, ERA-EDTA and national specialty organisations (USA, UK, Australia, European).

\section{Data extraction}

Data will be extracted from papers included in the scoping review using the draft data extraction tools listed in online supplementary tables $\mathrm{S} 2-5$ by two independent reviewers, and entered into spreadsheets. The data extracted will include specific details about the populations, concept, context, and study methods of significance to the scoping review question and specific objectives. Any disagreements that arise between the reviewers will be resolved through discussion, or with opinion from a third reviewer. When required, authors of papers will be contacted if possible to request missing or additional data. The draft data extraction tool will be modified and revised as necessary during the process of extracting data from each included study. Modifications will be detailed in the full scoping review report.

\section{Data mapping/analysis/synthesis and presentation of the results}

The extracted data will be presented in diagrammatic or tabular form that is relevant to the objectives and scope of this scoping review. We will summarise the information separately for each question that forms the basis of this scoping review.

For questions 1 and 2 (A), we will present in tabular form the various factors reported to influence prognosis/survival and quality of life, respectively, detailing the number of studies for each such factor, the number of patients studied, the settings, and provide comment about the generalisability of the findings.

For information addressing question 2 (B) - 'the lived experience of dialysis'; and question 3-'information needs for the shared decision-making process', the findings will be displayed in tabular and diagrammatic form, 
and in addition, we will use N-Vivo qualitative research software (QSR International, Australia) to synthesise the various themes identified.

A narrative summary will accompany the tabulated and/or charted results and will describe how the results relate to the primary questions around shared decisionmaking in the older patient with advanced renal disease. We anticipate that the identification of information needs will also guide the organisation of the information collected.

\section{Ethics and dissemination}

Ethical approval for the conduct of this study will not be required as this research only includes analysis of previously collected data. Results will be disseminated through academic journals, conferences and seminars. We will attempt to publish our findings in international open-access, peer-reviewed medical journals so that they are freely available.

\section{CONCLUSION}

The older patient with renal disease is different from younger counterparts on several counts-comorbidity burden, disease progression, survival, outcomes with therapy and considerations that influence quality of life. A 'one-size-fits-all' approach to counselling and prescribing renal replacement therapy cannot be recommended. The scoping review proposed will attempt to synthesise the disparate pieces of information available, and to be a resource for clinicians advising such older patients.

\section{Twitter Follow Rajesh Raj @kidneymedic and Mai Frandsen @FrandsenMai}

Contributors RR is the primary and corresponding author and was responsible for initial discussion and the first and all subsequent drafts. $\mathrm{KDKA}, \mathrm{MF}$ and MJ were involved in the initial discussion and design of the study protocol. They contributed to the design of the work, and revised the drafts critically for content. All four authors approved the final version to be published. All authors agree to be accountable for all aspects of the work in ensuring that questions related to the accuracy or integrity of any part of the work are appropriately investigated and resolved.

\section{Competing interests None declared.}

Provenance and peer review Not commissioned; externally peer reviewed.

Data sharing statement RR, MF, KDKA and MJ are the four authors of this scoping review protocol. The data used for creating this protocol were obtained jointly by the authors from readings of published literature. These data continue to be freely available to the authors via the library access provided by the University of Tasmania. The scoping review will collect articles to be considered for inclusion in the review. All bibliographic lists, full-text articles, data extraction tables, summary reports and the final written article will be stored on servers of the University of Tasmania, protected by user id/password access for all four researchers. Thus, all four researchers will have free and continued access to all the data collected and synthesised for this protocol development and the ensuing scoping review.

Open Access This is an Open Access article distributed in accordance with the Creative Commons Attribution Non Commercial (CC BY-NC 4.0) license, which permits others to distribute, remix, adapt, build upon this work noncommercially, and license their derivative works on different terms, provided the original work is properly cited and the use is non-commercial. See: http:// creativecommons.org/licenses/by-nc/4.0/

\section{REFERENCES}

1. ANZDATA Registry. 38th Report, Chapter 1: incidence of end stage kidney disease. Adelaide, Australia: Australia and New Zealand Dialysis and Transplant Registry, 2016.

2. Murtagh FE, Marsh JE, Donohoe P, et al. Dialysis or not? A comparative survival study of patients over 75 years with chronic kidney disease stage 5. Nephrol Dial Transplant 2007;22:1955-62.

3. Brown MA, Collett GK, Josland EA, et al. CKD in elderly patients managed without dialysis: survival, symptoms, and quality of life. Clin J Am Soc Nephrol 2015;10:260-8.

4. Da Silva-Gane M, Wellsted D, Greenshields H, et al. Quality of life and survival in patients with advanced kidney failure managed conservatively or by dialysis. Clin J Am Soc Nephrol 2012;7:2002-9.

5. Chandna SM, Da Silva-Gane M, Marshall C, et al. Survival of elderly patients with stage 5 CKD: comparison of conservative management and renal replacement therapy. Nephrol Dial Transplant 2011;26:1608-14

6. Schell JO, Da Silva-Gane M, Germain MJ. Recent insights into life expectancy with and without dialysis. Curr Opin Nephrol Hypertens 2013;22:185-92.

7. Morton RL, Turner RM, Howard K, et al. Patients who plan for conservative care rather than dialysis: a national observational study in Australia. Am J Kidney Dis 2012;59:419-27.

8. Brown MA, Crail SM, Masterson R, et al. ANZSN renal supportive care guidelines 2013. Nephrology 2013;18:401-54.

9. Renal Physicians Association. Clinical Practice Guideline on Shared Decision-Making in the Appropriate Initiation of and Withdrawal from Dialysis. 2nd edn. Rockville, Maryland, USA: Renal Physicians Association, 2010

10. Légaré $\mathrm{F}$, Ratté $\mathrm{S}$, Stacey $\mathrm{D}$, et al. Interventions for improving the adoption of shared decision making by healthcare professionals. In: Légaré F, ed. Cochrane database of systematic reviews. Chichester, UK: John Wiley \& Sons, Ltd, 2010. 9-11.

11. Elwyn G, Frosch D, Thomson R, et al. Shared decision making: a model for clinical practice. J Gen Intern Med 2012;27:1361-7.

12. Kee F. Stewardship or clinical freedom? Variations in dialysis decision making. Nephrol Dial Transplant 2000;15:1647-57.

13. Schell JO, Cohen RA. A communication framework for dialysis decision-making for frail elderly patients. Clin J Am Soc Nephrol 2014:9:2014-21.

14. Morton RL, Snelling P, Webster AC, et al. Dialysis modality preference of patients with CKD and family caregivers: a discrete-choice study. Am J Kidney Dis 2012;60:102-11.

15. Morton RL, Snelling P, Webster AC, et al. Factors influencing patient choice of dialysis versus conservative care to treat end-stage kidney disease. Can Med Assoc J 2012;184:E277-83.

16. The Joanna Briggs Institute. Joanna Briggs Institute Reviewers' Manual: Methodology for JBI Scoping Reviews. The Joanna Briggs Institute, 2015

17. Orimo H. [Reviewing the definition of elderly]. Nihon Ronen Igakkai Zasshi 2006;43:27-34. 